\title{
Síndrome de Burnout en médicos residentes de un Hospital público de la ciudad de Areguá en el año 2020
}

\section{Burnout syndrome in residents of a public hospital in the city of Areguá in 2020}

\author{
Anthon Daniel Torres Romero (D) ${ }^{1}$, Ivana Araceli Alcaraz Romero $\mathbb{D}^{2}$
}

${ }^{1}$ Universidad Nacional de Asunción, Facultad de Ciencias Médicas, San Lorenzo, Paraguay.

2Universidad del Norte, Facultad de Ciencias de la Salud, Asunción, Paraguay.

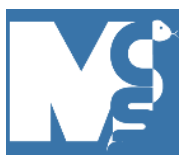

Recibido: $27 / 08 / 2020$

Revisado: 16/09/2020

Aceptado:21/09/2020

\section{Autor correspondiente}

Anthon Torres

Universidad Nacional de Asunción

San Lorenzo - Paraguay

antiel.ts.ro@gmail.com

\section{Conflictos de interés}

Los autores declaran no poseer conflictos de interés.

\section{Fuente de financiación}

Los autores no recibieron apoyo financiero para la investigación, autoría y/o publicación de este artículo.

Este artículo es publicado bajo una licencia de Creative Commons Reconocimiento 4.0 Internacional.

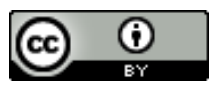

\section{RESUMEN}

Introducción: El estrés merece especial atención dentro de lo que sería la salud mental, teniendo en cuenta que estados prolongados de estrés podrían acarrear estados depresivos o de ansiedad severos. Las labores hospitalarias son uno de los trabajos de fuente innata para el estrés, este trabajo se enfocará en el estrés en médicos residentes. Objetivo: Determinar el síndrome de Burnout en médicos residentes de un hospital público de la ciudad de Areguá en el año 2020. Metodología: Estudio observacional descriptivo, de corte transversal, muestra no probabilístico por conveniencia, desde junio hasta agosto de 2020, de 24 encuestas, en el Hospital Nacional del Cáncer (Areguá). Se aplicó Cuestionario de Maslach Burnout Inventory- Healt Service Survey (MBI-HSS). Resultados: Se analizaron 24 encuestas. Edades comprendidas entre 24 y 34 años $(28,875 \pm 3,0405)$. Se reportan a 12 residentes con Síndrome de Burnout, equivalente al $54,2 \%$ de la muestra. Realización personal baja con 66,7\% (16); Agotamiento emocional alto 45,8\% (11); Despersonalización alta $50 \%$ (12). Conclusión: En la presente investigación se encontró que el 54,2\% de los residentes encuestados son categorizados con Síndrome de Burnout. Se resalta la investigación de Jacome et al donde el $47,5 \%$ de los residentes fueron categorizados con Síndrome de Burnout; mientras que, en el estudio de Estigarribia et al se encontró una baja prevalencia del Síndrome de Burnout, equivalente al 22\%; esta diferencia se puede deber al instrumento que fue utilizado en ambos estudios, tratándose del MBI..

Palabras clave: Agotamiento Psicológico; Estrés Psicológico; Síndrome de Burnout

\section{ABSTRACT}

Introduction: Stress deserves special attention within what would be mental health, taking into account that prolonged states of stress could lead to severe depressive or anxiety states. Hospital work is one of the innate source jobs for stress, this paper will focus on stress in medical residents. Objective: To determine the Burnout Syndrome in medical residents of a public hospital in the city of Areguá in the year 2020. Method: Descriptive, cross-sectional, non-probabilistic observational study from June to August 2020, of 24 surveys, at the National Cancer Hospital (Aregua). Maslach Burnout Inventory - Healt Service Survey (MBI-HSS) questionnaire was applied. Results: 24 surveys were analyzed. Ages between 24 and 34 years $(28,875 \pm 3,0405)$. Twelve residents with Burnout Syndrome were reported, equivalent to $54.2 \%$ of the sample. Low personal realization with $66,7 \%$ (16); high emotional exhaustion 45,8\% (11); high depersonalization $50 \%$ (12). Conclusion: In the present research it was found that $54.2 \%$ of the residents surveyed are categorized with Burnout Syndrome. Jacome et al's research is highlighted where $47,5 \%$ of the residents were categorized with Burnout Syndrome; while, in the study of Estigarribia et al a low prevalence of Burnout Syndrome was found, equivalent to $22 \%$; this difference can be due to the instrument that was used in both studies, being the MBI.

Keywords: Burnout; Psychological; Stress; Psychological; Burnout Syndrome 


\section{INTRODUCCIÓN}

El estrés se considera, por la Organización Mundial de la Salud, como una de las enfermedades de gran riesgo en el siglo XXI que llega a afectar al $4,4 \%$ de la población mundial (1). El estrés generalmente se encuentra asociado con el ámbito laboral (2). Es por esto que el riesgo de agotamiento aumenta significativamente en ciertas ocupaciones, especialmente para los trabajadores de la salud (3). Ya que afecta homeostasis y desencadena múltiples problemas de salud y de rendimiento en el trabajo (4).

Al conjunto de manifestaciones psicológicas e incluso físicas cuyo origen reside en la presión que sufren los profesionales al trabajar en relación directa con personas y que se encuentran sometidos a estresores laborales, se lo llama Síndrome de Burnout (5).

En un campo más específico, se encuentran los médicos residentes, estos se hallan bajo la presión extra que sugiere el hecho de ser estudiantes y trabajadores a la vez. El nivel de estrés puede sobrepasar su capacidad de afrontamiento y poner en riesgo o alterar su salud psicológica (6). Así como afectar su relación con los pacientes $(7,8)$.

El interés del estudio del Síndrome de Burnout se evidencia en diferentes países que determinan cifras del síndrome en los profesionales de la salud que van desde el $31 \%$ hasta un 42,2 \% (9). En la República del Paraguay ya se registran investigaciones que abarcan distintos enfoques para el mismo síndrome en servicios específicos, hospitales e incluso en universitarios (1012).

La finalidad de este trabajo es la de poner en evidencia el nivel de estrés a los que son sometidos los médicos residentes del Instituto Nacional del Cáncer; además de aportar una fuente informativa y de conocimiento de la situación actual de los profesionales.

\section{METOdOLOGÍA}

Se realizó un estudio observacional descriptivo, de corte transversal desde junio hasta agosto de 2020, en el Instituto Nacional del Cáncer (Areguá), Paraguay. Se incluyeron en el estudio a médicos residentes de los servicios del Instituto Nacional del Cáncer, con edades comprendidas entre 24 a 34 años. La selección de la muestra se realizó a través de técnicas no probabilísticas, por conveniencia.

Se realizó el cálculo de tamaño de muestra mediante la fórmula de proporción poblacional. La muestra de residentes necesarios para el estudio fue $=24$, considerando una población de $\mathrm{N}=25$ residentes, confianza del $95 \%(Z=1,96)$, margen de error $E=5 \%$ y proporción de éxito $\mathrm{p}=\mathrm{q}$. Se midieron 28 ítems repartidos en 3 categorías: sociodemográficas (edad, sexo, estado civil, año de residencia), desgaste temporal (guardias por semana, horas de guardia por semana) y síndrome de burnout.

Se utilizó el Cuestionario de Maslach Burnout Inventory- Healt Service Survey (MBI-HSS), adaptado para la población hispanohablante que consta de 22 preguntas con formato Likert de 7 alternativas: 0Nunca, 1- Alguna vez al año o menos, 2-Una vez al mes o menos, 3- Algunas veces al mes, 4- Una vez a la semana, 5- Varias veces a la semana, 6- Diariamente. Su puntaje máximo es de 132 puntos y consta de 3 subescalas: Agotamiento Emocional, Despersonalización, Realización Personal. El instrumento posee un alfa de Cronbach de 0.74 que indica una buena confiabilidad (13).

Subescala de Agotamiento Emocional (AE). Consta de 9 preguntas. Valora la vivencia de estar exhausto emocionalmente por las demandas del trabajo. Puntuación máxima 54, cuanto mayor es la puntuación en esta subescala mayor es el agotamiento emocional, se categoriza de la siguiente manera: bajo [0-18], intermedio [19-26], alto [27-54].

Subescala de Despersonalización. Está formada por 5 ítems. Valora el grado en que cada uno reconoce actitudes de frialdad y distanciamiento. La puntuación máxima es de 30 puntos, cuanto mayor es la puntuación en esta subescala mayor es la despersonalización, se categoriza de la siguiente manera: bajo [0-6], intermedio [7-9], alto [10-30].

Subescala de Realización Personal. Se compone de 8 ítems. Evalúa los sentimientos de autoeficacia y realización personal en el trabajo. La puntuación máxima es de 48 puntos, y cuanto mayor es la puntuación en esta subescala mayor es la realización personal, se categoriza de la siguiente manera: bajo [029], intermedio [30-39], alto [40-48].

Aunque no hay puntaciones de corte a nivel clínico para medir la existencia o no de burnout, puntuaciones altas en Agotamiento Emocional y Despersonalización y baja en Realización Personal definen el síndrome. Para el procesamiento de la información se creó una base de datos en el software Microsoft Office Excel 2013. Se excluyeron todas las encuestas incompletas, en blanco o mal llenadas. Posteriormente, se exportó la base de datos al programa PSPP (1.0.1-g818227), donde se realizó el análisis de los datos.

Las variables cualitativas se presentan con frecuencias y porcentajes. Las variables cuantitativas con distribución normal se presentan con medias y 
desviación estándar. El presente estudio fue realizado siguiendo los lineamientos establecidos en la declaración de Helsinki, mediante un consentimiento informado proporcionado previo a la realización de la encuesta y manteniendo el anonimato.

\section{RESULTADOS}

Fueron analizados las encuestas de 24 médicos residentes de un Hospital Público en Areguá. En la
Tabla 1 se puede apreciar que predomina el sexo Femenino, con una frecuencia de 16 personas, lo que equivale al $66,7 \%$ del total de encuestados. La mayoría de los médicos encuestados afirman un estado civil de soltero, con una frecuencia de 20 personas, equivalente al 83,3 \% de la muestra. Los mismos realizan con mayor frecuencia ente 2 y 3 guardias por semana.

TABLA 1. VARIABLES CUALITATIVAS DE LOS MÉDICOS RESIDENTES DEL INSTITUTO NACIONAL DEL CÁNCER EN AREGUA, 2020 $(n=24)$.

\begin{tabular}{|c|c|c|c|}
\hline \multicolumn{2}{|l|}{ Variable } & Frecuencia & Porcentaje \\
\hline \multirow[t]{2}{*}{ Sexo } & Hombre & 8 & 33,3 \\
\hline & Mujer & 16 & 66,7 \\
\hline \multirow[t]{3}{*}{ Estado civil } & Casado & 3 & 12,5 \\
\hline & Soltero & 20 & 83,3 \\
\hline & Unión libre & 1 & 4,2 \\
\hline \multirow[t]{4}{*}{ Años de residencia } & Primero & 4 & 16,7 \\
\hline & Segundo & 7 & 29,2 \\
\hline & Tercero & 5 & 20,8 \\
\hline & Cuarto & 8 & 33,3 \\
\hline \multirow[t]{4}{*}{ Guardias por semana } & 0 & 5 & 20,8 \\
\hline & 1,0 & 3 & 12,5 \\
\hline & 2,0 & 8 & 33,3 \\
\hline & 3,0 & 8 & 33,3 \\
\hline \multirow[t]{2}{*}{ Síndrome de Burnout } & Sí & 13 & 54,2 \\
\hline & No & 11 & 45,8 \\
\hline \multirow[t]{3}{*}{ Realización personal } & Alto & 3 & 12,5 \\
\hline & Medio & 5 & 20,8 \\
\hline & Bajo & 16 & 66,7 \\
\hline \multirow[t]{3}{*}{$\begin{array}{l}\text { Agotamiento } \\
\text { emocional }\end{array}$} & Alto & 11 & 45,8 \\
\hline & Medio & 2 & 8,3 \\
\hline & Bajo & 11 & 45,8 \\
\hline \multirow[t]{3}{*}{ Despersonalización } & Alto & 12 & 50,0 \\
\hline & Medio & 2 & 8,3 \\
\hline & Bajo & 10 & 41,7 \\
\hline
\end{tabular}

En la Tabla 2 se puede apreciar que la edad media de dichos profesionales fue $28,8 \pm 3,04$. Los encuestados afirman de 18 a 140 horas de servicio a la semana con una media de $75,67 \pm 31,18$. La puntuación máxima obtenida mediante el cuestionario de MBI-HSS fue de 94 puntos y la puntuación mínima fue de 11 con una media de $51,54 \pm 28,97$. Respecto al desgaste laboral, en la Tabla 3, los residentes de segundo año presentan una mayor frecuencia de guardas por semana, así como una media de 119,6 $\pm 11,9$ horas. 
TABLA 2. VARIABLES CUANTITATIVAS DE LOS MÉDICOS RESIDENTES DEL INSTITUTO NACIONAL DEL CÁNCER EN AREGUA, 2020 $(\mathrm{N}=\mathbf{2 4})$

$\begin{array}{lcccc} & \text { Vánimo } & \text { Máximo } & \text { Media } & \text { Desvió Estándar } \\ \text { Edad } & 24 & 34 & 28,87 & 3,04 \\ \text { Horas de Guardia por semana } & 18 & 140 & 75,67 & 31,18 \\ \text { Síndrome de Burnout } & 11 & 94 & 51,54 & 28,97 \\ \text { Realización personal } & 5 & 46 & 22,13 & 13,37 \\ \text { Agotamiento emocional } & 2 & 39 & 20,25 & 13,18 \\ \text { Despersonalización } & 0 & 20 & 9,17 & 7,54\end{array}$

TABLA 3. DESGASTE LABORAL DE LOS MÉDICOS RESIDENTES DEL INSTITUTO NACIONAL DEL CÁNCER EN AREGUA, 2020 (N=24)

\begin{tabular}{lccccc}
\hline Año de residencia & $\begin{array}{c}\text { Guardias } \\
\text { semanales }\end{array}$ & Frecuencia & Porcentaje & $\begin{array}{c}\text { Media (horas por } \\
\text { semana) }\end{array}$ & $\begin{array}{c}\text { Desviación típica } \\
\text { Primer año }\end{array}$ \\
\hline \multirow{2}{*}{ Segundo año } & 3 & 1 & 25 & 60,0 & \\
& 0 & 3 & 75 & 72,0 & 46,8 \\
Tercer año & 2 & 1 & 14,28 & 55,0 & \\
& 3 & 5 & 14,28 & 84,0 & 11,9 \\
Cuarto año & 0 & 1 & 71,44 & 119,6 & \\
& 1 & 1 & 20 & 55,0 & 12,6 \\
& 2 & 3 & 60 & 55,0 & 8,1 \\
& 0 & 3 & 37,5 & 68,3 & 25,5 \\
\end{tabular}

La aparición de síndrome de Burnout es más frecuente en residentes que no cumplen con ninguna guardia, ya que el $80 \%$ de los que no tienen guardia reportan síndrome de Burnout. Así mismo el 66,6 \% de los que tienen una guardia, el $37,5 \%$ de los que tienen dos guardias y el $50 \%$ de los que tienen tres guardias.

\section{DISCUSIÓN}

En el presente estudio se tuvo como principal objetivo determinar el nivel de síndrome de Burnout en médicos residentes del Instituto Nacional del Cáncer de Paraguay. En este punto, es propicio señalar que no se encuentran publicaciones de trabajos de investigación que determinen el nivel de este síndrome en dicho instituto.

En cuanto a las variables sociales que se consideraron en el presente estudio, la mayoría son del sexo femenino, con edades comprendidas entre 24-34 años, con una media de 28,87 $\pm 3,04$ y estado civil predominantemente soltero. Los presentes resultados concuerdan con los de Marecos-Bogado donde se estudia un rango etario similar entre 23-34 años con una media de $28,86 \pm 1,89$ y donde predomina el sexo femenino, así como los residentes encuestados afirman estar en la categoría de solteros en su estado civil (14).
El $75 \%$ de los residentes de primer año tienen síndrome de Burnout, el 28,58 \% de los residentes de segundo año, el $60 \%$ de los residentes de tercer año y el $62,5 \%$ de los residentes de cuarto año.

A su vez, se encuentran resultados sociales similares en investigaciones de dicho síndrome en residentes del Paraguay, donde el rango etario se comprende entre 26-31 años y predomina el sexo femenino y solteros como estado civil (12); así como otro estudio donde la edad media de residentes de un hospital paraguayo fue de 27,65 $\pm 2,37$ años, principalmente del sexo femenino y solteros en su estado civil (15). Sin embargo, el estudio de Estigarribia y colaboradores registra un rango etario más amplio, se incluye a residentes de 25 55 años con una media de $35,8 \pm 7,4$ y predomina el sexo masculino (16).

Respecto al desgaste laboral, en la presente investigación enfocada en los residentes del Instituto Nacional del Cáncer, no se discrimina por especialidades, por ende, algunos residentes cuentan con 3 guardias por semana, mientras que otros no cuentan con ninguna, como es el caso para la especialidad de Patología. De la misma manera, se 
registran trabajos donde se hace presente dicha discriminación entre las variables con el fin de definir la presencia exacta del Síndrome de Burnout en varios servicios (14); mientras que otras investigaciones dirigen su enfoque a servicios hospitalarios paraguayos específicos como es el caso de Medicina Familiar (12).

A su vez, los residentes que sí practican guardias, registran una actividad media de más de 70 horas por semana. La investigación de Larrea Pinto indica una cantidad similar, ya que los residentes cumplen con aproximadamente 80 horas semanales de guardia (17). Mientras que en el trabajo de Medina et al se registra una actividad de apenas 44 horas semanales de guardia médica (18); sin embargo, el estudio de Zambrano Toala afirma que el registro de médicos residentes con hasta 120 horas semanales (19). Dicho escenario puede ser propicio para el desarrollo de síndrome de Burnout.

En la presente investigación se encontró que el 54,2 \% de los residentes encuestados son categorizados con Síndrome de Burnout. Se resalta la investigación de Jacome et al donde el $47,5 \%$ de los residentes fueron categorizados con Síndrome de Burnout (20); esta similitud se puede deber a que en ambos estudios fue utilizado el Maslach Burnout Inventory Human Services Survey para medir el Síndrome de Burnout en profesionales de la salud.

La investigación de Arayago y colaboradores realizada en médicos residentes de anestesiología evidencia que el 73,3 \% de dicho personal cuenta con Síndrome de Burnout (21); este resultado, que sobrepasa a lo indicado en el presente trabajo, se puede deber al instrumento que utilizaron (Mashlac Burnout Inventory-MBI) y al servicio que enfocaron su estudio (anestesiología). Sin embargo, en el estudio de Estigarribia y colaboradores se encontró una baja prevalencia del Síndrome de Burnout, equivalente al 22 $\%$ (16); mientras que en el trabajo de Marecos-Bogado se encontró un $24 \%$ de prevalencia para dicho síndrome (14); esta diferencia se puede deber al instrumento que fue utilizado en ambos estudios, tratándose del MBI.

En el presente estudio se encontró una prevalencia del $66,7 \%$ en residentes con Baja de Realización Personal. Lo cual coincide con Marecos-Bogado donde encontraron una puntuación baja en $42,3 \%$ de los médicos residentes (14). Este resultado discrepa con el de Jacome y colaboradores debido a que, en dicho trabajo, se registra una realización personal alta, equivalente al $50 \%$ de los médicos residentes (20).

Se encontró un igual porcentaje de residentes con la categorización Alta y Bajo para las Afecciones Emocionales ( $45,8 \%$ en ambos casos). Estos resultados coinciden con los de Jacome et al donde también se registra un nivel Alto y Bajo, lo que equivale a $42,5 \%$ de su muestra (20). Lo cual discrepa del resultado obtenido en la investigación de Mareco-Bogado donde se registra un $58,7 \%$ de los residentes con alto nivel de Afecciones Emocionales, mientras un $13,5 \%$ para el nivel bajo del mismo factor (14).

En esta investigación, se detectó un alto nivel de Despersonalización en los médicos residentes (50 \%). El mismo resultado, coincide con lo obtenido por Marecos-Bogado, donde se destaca una alta puntuación en despersonalización, equivalente al 78,8 $\%$ de los médicos residentes (14). Este resultado no coincide con los obtenidos por Jacome y colaboradores donde la mayoría de los encuestados obtuvieron un bajo nivel de despersonalización, equivalente al $55 \%$ de los residentes encuestados (20); esta discrepancia se puede deber a las características sociales y demográficas distintas para ambos estudios.

Es apropiado aclarar que este estudio tiene las siguientes limitantes: residentes no diagnosticados con sintomatología depresiva, estrés laboral, así como los factores que predisponen a la inestabilidad del estado de ánimo. Sin embargo, esta investigación actualiza y amplía la bibliografía existente en el Paraguay sobre el Síndrome de Burnout, sin mencionar que es el primer estudio de dicho síndrome en el Instituto Nacional del Cáncer ubicado en Areguá.

Respecto al objetivo general de la investigación, se demuestra presencia del síndrome de Burnout en los médicos residentes del Instituto Nacional del Cáncer de Areguá. Esto deja en evidencia la necesidad de tomar medidas de acción para solventar la carga de estrés en dichos profesionales antes de que dicho síndrome genere otras patologías psicológicas; de esta manera, lograr optimizar su tiempo y mejorar la relación médico-paciente. Se recomiendan los siguientes puntos: 1) Estudiar con más detalles las causas que exponen a los residentes a padecer estrés laboral y en consecuencia desarrollen Síndrome de Burnout, 2) Expandir la muestra del estudio de tal manera que permita analizar y evidenciar la relación entre otros hospitales públicos.

\section{REFERENCIAS}

1. Organización Panamericana de la Salud. Depresión y otros trastornos mentales comunes. Estimaciones sanitarias mundiales. WHO/MSD/MER/2017.2; 2017.

2. Gil-Monte PR. Validez factorial de la adaptación al español del Maslach Burnout Inventory-General Survey. Salud Pública México .2002;44 (1):33-40.

3. Rössler W. Stress, burnout, and job dissatisfaction in mental health workers. Eur Arch Psychiatry Clin Neurosci .2012;262 Suppl 2:S65-69. https://dx.doi.org/10.1007/s00406-0120353-4 
4. Mejía IAO, Morales SC, Orellana PC, Lorenzo AF. Efecto de las actividades físicas en la disminución del estrés laboral. Revista Cubana de Medicina General Integral .2017;33 (3):342-51.

5. Caballero Domínguez C, Hederich C, Palacio Sañudo J. Academic burnout: Delineation of the syndrome and factors associated with their emergence. Rev Latinoam Psicol .2010;42:131-46.

6. Schwenk TL, Davis L, Wimsatt LA. Depression, stigma, and suicidal ideation in medical students. JAMA .2010;304 (11):1181-90. https://dx.doi.org/10.1001/jama.2010.1300

7. Shanafelt TD, Bradley KA, Wipf JE, Back AL. Burnout and self-reported patient care in an internal medicine residency program. Ann Intern Med .2002;136 (5):358-67. https://dx.doi.org/10.7326/0003-4819-136-5-200203050$\underline{00008}$

8. Vivanco MEV, León CDS, Rivera RPM, Ortega ERNE. Síndrome de burnout en profesionales de la salud en instituciones públicas y privadas: un análisis en la provincia de Loja-Ecuador. Rev Bol Redipe .2018;7(11):179-94.

9. Grau A, Flichtentrei D, Suñer R, Font-Mayolas S, Ferrer MP i, Braga F. El burnout percibido o sensación de estar quemado en profesionales sanitarios: prevalencia y factores asociados. Inf Psicològica .2008(91-92):64-79.

10. Acosta FV, Abente SV, Galeano IO. Frecuencia del síndrome de Burnout en residentes de Medicina Interna del Hospital de Clínicas. Rev Virtual Soc Paraguaya Med Interna .2020:72-79.

11. Rojas-Melgarejo J, Mereles-Oviedo F, Barrios I, Torales J. Síndrome de Burnout en estudiantes de medicina de Santa Rosa del Aguaray: Burnout Syndrome in medical students of Santa Rosa del Aguaray. Med Clín Soc .2017;1 (1):26-30.

12. Szwako Pawlowicz AA. Relaciòn entre funcionalidad familiar y Sindrome de Burnout en residentes de medicina familiar. Rev Salud Publica Parag .2014;4(1):21-26-26.

13. Olivares-Faúndez V, Mena-Miranda L, Macía-Sepúlveda F, Jélvez-Wilke $C$. Validez factorial del Maslach Burnout Inventory Human Services (MBI-HSS) en profesionales chilenos. Univ Psychol .2014;13 (1):145-60. https://dx.doi.org/10.11144/Javeriana.UPSY13-1.vfmb

14. Marecos-Bogado S, Moreno M. Asociación entre Síndrome de Burnout y funcionalidad familiar en médicos residentes: Association between Burnout Syndrome and family functionality in resident physicians. Med Clín Soc .2018;2 (2):54-64.

15. Alfonso Recalde SS, Ferreira Gaona MI, Díaz Reissner CV. Síndrome de burnout en residentes de especialidades médicas. Hospital Central Dr. Emilio Cubas del IPS. Eureka .2015;12(1):25-34.

16. Estigarribia G, Rodriguez LCA, Guerrero C. Prevalencia de Síndrome de Burnout en Médicos del Hospital Regional de Coronel Oviedo, 2017. Rev Científica Fac Cienc Medicas Univ Nac Caaguazu .2018;1(2):44-53.

17. Larrea Pinto MA. Síndrome de Burnout en los médicos residentes del HNAAA. Univ Nac Pedro Ruiz Gallo.2019.

18. Medina ML, Medina MG, Gauna NT, Molfino L, Merino LA. Prevalencia del síndrome de burnout en residentes de Pediatría de un hospital. Investig En Educ Médica .2017;6 (23):160-8. https://dx.doi.org/10.1016/j.riem.2017.03.004

19. Zambrano Toala JR. Burnout Syndrome in Resident Doctors. Rev San Gregor.2019(33):102-13.

https://dx.doi.org/10.36097/rsan.v1i33.966

20. Jacome SJ, Hurtado AFV, García CP, Duque IL. Prevalencia del síndrome de Burnout en residentes de especialidades médicas. Rev Cuid.2019;10(1):10.

21. Arayago R, González A, Limongi M, Guevara H. Síndrome de Burnout en residentes y especialistas de anestesiología. Rev SalusUC.2016;20 (1):13-21. 